\title{
Melhoria da saúde pública e dos sistemas de saúde por meio de política informada por evidências nas Américas*
}

\author{
Tomás Pantoja ${ }^{1}$, Jorge Barreto ${ }^{2}$ y Ulysses Panisset ${ }^{3}$
}

\author{
A tradução do conhecimento em políticas e práticas pode melhorar a saúde pública e os \\ sistemas de saúde nas Américas, afirmam Tomás Pantoja e colegas
}

A estratégia de formulação de políticas informadas por evidências - na qual as decisões políticas são informadas pelo uso sistemático e transparente de evidências - foi objeto de grande atenção internacional no início do milênio. O movimento foi impulsionado pelo Relatório mundial sobre conhecimentos para melhorar a saúde de 2004 e pelas declarações emitidas nas Reuniões de Cúpula Ministerial de Pesquisa em Saúde na Cidade do México em 2005 e em Bamaco, Mali, em 2008, ambas convocadas pela Organização Mundial da Saúde. ${ }^{12}$ Mais recentemente, a Agenda 2030 das Nações Unidas para o desenvolvimento sustentável formulou 17 objetivos para o desenvolvimento global, e as políticas informadas por pesquisa serão essenciais.

Nesse contexto, a Organização PanAmericana da Saúde/Organização Mundial da Saúde (OPAS/OMS) elaborou sua "Política de Pesquisa para a Saúde" em 2009. ${ }^{3}$ Essa política salientou a necessidade de pertinência e padrões mínimos de qualidade nas pesquisas realizadas para informar a política de saúde. Além disso, definiu a conjuntura necessária para produzir pesquisas impactantes,

\footnotetext{
* Tradução oficial para o português realizada pela Organização Pan-Americana da Saúde. Em caso de discrepância, prevalecerá a versão original em inglês.
}

que é a governança efetiva da pesquisa e pessoal capacitado atuando em redes de parceria. ${ }^{3} \mathrm{O}$ objetivo político de alcançar um impacto positivo na saúde por meio da pesquisa depende do estabelecimento de comunicação construtiva entre os diferentes interessados diretos que financiam, produzem e usam a pesquisa. Isso pressupõe o empoderamento de organizações da sociedade civil para que definam prioridades de pesquisa e disponibilizem os resultados das pesquisas em formatos apropriados para diferentes públicos.
O objetivo deste artigo é examinar os desafios atuais da pesquisa em saúde e descrever iniciativas de sucesso implementadas nas Américas com o objetivo de ter um impacto positivo na saúde da população.

\section{PRINCIPAIS DESAFIOS}

Os pesquisadores identificaram dificuldades e limitações que prejudicam os esforços para associar a pesquisa à ação política no âmbito nacional. ${ }^{4}$ Esses problemas podem ser agrupados em três áreas

\section{MENSAGENS-CHAVE}

- Muitos formuladores de políticas nas Américas reconhecem a importância de usar a pesquisa para informar decisões tomadas em seus sistemas de saúde.

- Entretanto, a tradução do conhecimento é muito mais aceita na formulação de políticas clínicas e de saúde pública que no campo dos sistemas de saúde.

- Nas Américas, o crescimento e a consolidação de sistemas nacionais de pesquisa em saúde para gerar evidências de pesquisa têm sido desiguais e baseados em iniciativas isoladas de capacitação.

- Alguns países criaram uma infraestrutura formal para implementar esforços de tradução da pesquisa no âmbito nacional.

- Apesar disso, na maioria dos casos, as atividades são desenvolvidas como projetos pontuais sem financiamento sustentável após o ciclo de vida do projeto específico.

\footnotetext{
Professor associado, Departamento de Medicina Familiar, Pontificia Universidad Católica de Chile, Santiago, Chile. $₫$ tpantoja@med.puc.cl

2 Pesquisador, Fundação Oswaldo Cruz (Fiocruz), Brasília, Brasil.
} 
principais: o clima sociopolítico para uso da pesquisa; o processo de produção da pesquisa; e a tradução dos resultados da pesquisa. Com frequência, a pesquisa é subvalorizada, vista como irrelevante ou de uso muito difícil para informar a formulação de políticas.

Embora os formuladores de políticas em muitos países nas Américas reconheçam os benefícios do uso da pesquisa para orientar decisões destinadas a melhorar os sistemas de saúde, as ações atuais ainda são pálidas em comparação com os avanços obtidos pelo movimento da medicina baseada em evidências. Os produtores e usuários de pesquisa ainda lutam para desenvolver os fundamentos científicos da pesquisa em políticas e sistemas de saúde. ${ }^{5}$ Além disso, o uso sistemático da pesquisa nos processos decisórios no sistema de saúde não foi formalmente estabelecido nos sistemas de formulação de políticas. Na atualidade, isso depende muito da bagagem dos responsáveis pelas decisões e de suas relações pessoais com pesquisadores e profissionais de saúde. ${ }^{6}$

As Américas, incluindo a região do Caribe, produzem $46 \%$ da pesquisa em saúde pública no mundo -, porém a maior parte $(37 \%)$ vem dos Estados Uni$\operatorname{dos}^{7}$ e nem sempre tem pertinência direta para os Estados Membros da OPAS. ${ }^{8}$ É necessária uma abordagem mais sistemática para estabelecer prioridades e financiar pesquisas pertinentes e úteis para as Américas. Sistemas nacionais de pesquisa em saúde, pessoas, instituições e atividades que produzam pesquisa de alta qualidade, para promover, restaurar e manter o estado de saúde das populações, são uma maneira de assegurar que a pesquisa corresponda às necessidades de saúde pública nacionais. Entretanto, o crescimento e a consolidação desses sistemas nas Américas foi desigual e baseado em iniciativas de capacitação individuais ou isoladas. ${ }^{9}$

Em 2005, a Assembleia Mundial da Saúde instou os países a criarem infraestruturas nacionais para promover políticas baseadas em evidências e sistemas de saúde pública e de atenção à saúde baseados em evidências. ${ }^{10} \mathrm{Na}$ prática, essa infraestrutura consiste em programas, intervenções e ferramentas que disseminam e facilitam o acesso a informações de pesquisa, promovem o intercâmbio de conhecimento entre interessados diretos e usam evidências para informar as organizações de atenção à saúde e os sistemas de saúde. ${ }^{11}$ No entanto, atualmente muitos países nas Américas não dispõem dessa infraestrutura formal. ${ }^{12} \mathrm{Na}$ maioria dos casos, as atividades são desenvolvidas como projetos pontuais, sem financiamento sustentável após o ciclo de vida do projeto. Embora algumas iniciativas tenham promovido o diálogo entre produtores e usuários de pesquisas, a alta rotatividade de autoridades de saúde pública, e até mesmo de equipes técnicas dentro dos setores de saúde e tecnologia, prejudicam o desenvolvimento de atividades de tradução do conhecimento a longo prazo. ${ }^{12}$ Além disso, a falta de uma infraestrutura formal para coordenar os esforços nacionais para traduzir a pesquisa em políticas e promover o intercâmbio de experiências entre os países das Américas limitou a aprendizagem coletiva e o compartilhamento de recursos na área de tradução do conhecimento.

\section{INICIATIVAS BEM-SUCEDIDAS}

Não houve uma avaliação exaustiva das iniciativas de tradução do conhecimento nas Américas. Entretanto, há casos no Brasil, no Chile e no Peru de resposta aos desafios enfrentados ao tentar combinar a pesquisa à ação política. Os autores selecionaram esses estudos de caso com base em seu conhecimento direto sobre eles.

No Peru, nos últimos anos, o clima sociopolítico para uso da pesquisa melhorou graças ao trabalho de unidades de pesquisa não acadêmicas que promovem o uso de evidências de pesquisas no sistema de saúde. Depois de duas iniciativas em que resultados de pesquisas ajudaram a informar decisões políticas, o Ministério da Saúde do Peru criou processos formais para exigir evidências de pesquisas. As duas iniciativas concentraram-se nos efeitos abortivos da pílula anticoncepcional da manhã seguinte e no possível impacto de alimentos transgênicos sobre a saúde. ${ }^{13}$ Atualmente, há exigência de evidências de pesquisa para informar decisões sobre o orçamento de programas, a reformulação de programas de saúde nacionais e subnacionais, bem como a seleção de intervenções de saúde específicas a serem financiadas pelo ministério. Para responder à necessidade de evidências no Peru, criou-se, no Instituto Nacional de Saúde, a Unidade para Geração e Análise de Evidências em Saúde Pública. ${ }^{14}$ Além disso, esses processos formais foram ampliados para outras diretorias no Ministério da Saúde e iniciou-se a colaboração entre grupos que trabalham com diretrizes clínicas e avaliação de tecnologias em saúde.

No Chile, o Fundo Nacional de Pesquisa em Saúde (FONIS) é uma iniciativa custo-efetiva organizada para produzir pesquisas pertinentes que ajudem os responsáveis pelas decisões a corrigir os problemas no sistema de saúde do país. ${ }^{15}$ Desde 2003, o FONIS instituiu um fundo competitivo para financiamento de projetos nas áreas de efetividade das intervenções clínicas e de saúde pública; saúde ambiental e ocupacional; equidade no acesso à atenção à saúde; promoção da saúde e controle de fatores de risco; qualidade da atenção à saúde e satisfação do usuário com os serviços de saúde; e métodos para medir o impacto na saúde das políticas públicas implementadas por setores não relacionados com a saúde. Nos seus 10 primeiros anos, o FONIS financiou 300 projetos com custo total aproximado de 10 milhões de dólares $(7,5$ milhões de libras; 8,6 milhões de euros). As pesquisas financiadas pelo FONIS tiveram impacto na política nacional. As modificações da lei do tabaco no Chile, por exemplo, foram informadas por pesquisa financiada pelo FONIS. ${ }^{16} 17$

Em 2006, a OMS lançou sua Rede para Políticas Informadas por Evidências (EVIPNet) para enfrentar o desafio de traduzir as pesquisas em políticas nas Américas e no mundo. A EVIPNet desenvolve parcerias sustentáveis entre os formuladores de políticas, os pesquisadores e a sociedade civil para aumentar a capacidade dos países de elaborar políticas de saúde com uso de evidências de pesquisa. ${ }^{18}$ A OPAS lançou a EVIPNet nas Américas em 2007 e, ao final de 2013, havia equipes em 12 países. ${ }^{12}$ As equipes nacionais da EVIPNet formam a infraestrutura institucional necessária para traduzir os resultados das pesquisas em políticas efetivas. Os dois principais produtos das equipes nacionais da EVIPNet são as sínteses de evidências acessíveis e contextualizadas para política, que apresentam uma síntese da pesquisa de diferentes opções políticas, e os diálogos deliberativos, que são discussões estruturadas centradas em uma síntese de evidências e usadas para formular políticas. ${ }^{19}$ Até 2013, mais de 700 funcionários e especialistas dos países haviam participado de treinamento, elaborado 14 sínteses de evidências e realizado 10 diálogos deliberativos em oito países. ${ }^{12}$ 


\section{Quadro 1: A experiência da EVIPNet-Brasil}

Em 2016, a EVIPNet-Brasil publicou uma série de oito sínteses de evidências em saúde para política em áreas públicas prioritárias. As elevadas taxas de mortalidade infantil no norte e nordeste brasileiro impulsionaram esse esforço para usar evidências. As evidências mostraram a correlação direta existente entre a alta mortalidade infantil e a baixa qualidade da atenção durante o trabalho de parto e o parto, com profissionais da saúde insuficientemente capacitados que negligenciavam protocolos e diretrizes de atenção estabelecidos. Em Piripiri, município de poucos recursos, a pesquisa revelou que $60 \%$ das mortes de recém-nascidos eram evitáveis.

A EVIPNet-Brasil tratou dessa questão pela apresentação de opções de políticas informadas em evidências em parceria com o conselho de saúde local. Diálogos deliberativos levaram à seleção das opções com maior potencial de impacto, incluindo o planejamento local complexo de mais de 30 intervenções com base nas melhores evidências disponíveis. Esse trabalho ajudou a reduzir as taxas de mortalidade infantil em Piripiri, de 21 por 1.000 nascidos vivos em 2009, para 7 por 1.000 nascidos vivos em 2011.

Atualmente, a EVIPNet-Brasil está adaptando essas evidências em um modelo de política para que possa ser reproduzido em várias outras localidades brasileiras, onde grupos de trabalho locais responderão aos próprios problemas de saúde, prepararão sínteses de evidências e organizarão diálogos deliberativos sobre os temas que os afetam. Outras questões prioritárias de saúde pública são anemia falciforme, poluição do ar, cardiopatia congênita, tuberculose na população desabrigada, desenvolvimento da criança na primeira infância e mortes no trânsito.

O Brasil abriu o caminho na implementação bem-sucedida de atividades e produtos da EVIPNet (Quadro 1). ${ }^{20}$ Embora não seja a única iniciativa a responder ao desafio de traduzir pesquisas em políticas, a EVIPNet forneceu um modelo para organização de atividades em outros países nas Américas (Chile, por exemplo). ${ }^{21}$ Além disso, proporcionou oportunidades de campo para capacitação, ajudando tanto pesquisadores quanto responsáveis por decisões por meio da rede da iniciativa global EVIPNet.

\section{AGENDA EM ABERTO}

A fim de produzir pesquisas relevantes para informar a tomada de decisão em saúde, os países precisam adotar uma estratégia sistemática de fortalecimento dos sistemas nacionais de pesquisa em saúde. ${ }^{22}$ Isso demanda uma política ou um programa bem definido vinculado às prioridades nacionais de pesquisa em saúde; estruturas para coordenar e administrar a pesquisa; mecanismos sustentáveis de financiamento; e um conjunto específico de indicadores para monitoramento e avaliação. A OPAS ocupa boa posição para promover a adoção dessa estratégia nos países das Américas, aproveitando as experiências bem-sucedidas e conectando de maneira sistemática e coerente as iniciativas isoladas atuais.

Apesar de ter havido algum êxito na tradução do conhecimento nas Américas, $^{20,21}$ ainda é necessário fortalecer a infraestrutura de tradução da pesquisa na maioria dos países. Os países também precisam promover e apoiar a criação de equipes de tradução da pesquisa. Isso é crucial nos países em que foram realizadas algumas atividades de tradução, mas não se estabeleceu infraestrutura formal para colaboração regional ou global. Para além das questões de financiamento, o balanço dos êxitos e fracassos depois de 10 anos de esforço nessa área será uma prioridade a curto prazo. A OPAS está tentando responder aos desafios que prejudicam a tradução do conhecimento mediante a estreita colaboração com parceiros e o desenvolvimento de alianças estratégicas. Entre elas estão o trabalho com a Universidade McMaster, no Canadá, e o Centro Latino-americano e do Caribe de Informação em Ciências da Saúde, no Brasil; a disponibilização da Biblioteca Virtual em Saúde regional e do sistema integral de base de dados da literatura; e o trabalho com a Aliança para Pesquisa em Políticas e Sistemas de Saúde da OMS.

Não houve avaliação formal nas Américas do clima sociopolítico em relação ao uso de pesquisas para informar políticas, e os dados sobre esse tema estão dispersos em documentos políticos de diferentes países. Não houve também um esforço sistemático para reunir informações sobre as condutas predominantes relativas à tradução do conhecimento, como esforços feitos no Oriente Médio e em alguns países africanos. ${ }^{23} 24$ Esse tipo de informação possibilitaria a concepção de estratégias específicas. A OPAS está em posição privilegiada para mobilizar grupos de especialistas regionais que trabalham no campo e responder a esse problema.

\section{CONCLUSÕES}

No âmbito nacional, há várias dificuldades para vincular a pesquisa à ação de política. Os problemas estão relacionados ao clima sociopolítico relacionado ao uso de pesquisas; à produção de pesquisas relevantes; e à tradução dos resultados das pesquisas. A Política de Pesquisa para a Saúde da OPAS estabeleceu uma estrutura geral para a organização de iniciativas bem-sucedidas nas Américas. Durante a última década, houve avanços no estabelecimento de sistemas nacionais de pesquisa em saúde, bem como na disponibilidade de equipes nacionais competentes para identificar, avaliar, resumir e compilar evidências de pesquisa. Houve também avanços na definição de processos padronizados para o uso de evidências na formulação de políticas (por exemplo, processos da EVIPNet), com o apoio desses esforços por equipes de especialistas regionais. Entretanto, os sistemas de saúde da região ainda enfrentam obstáculos à geração de impacto por meio de pesquisa. A OPAS e sua Política de Pesquisa para a Saúde serão essenciais para definir os próximos passos para alcançar um impacto positivo na saúde por meio da pesquisa sobre sistemas de saúde nas Américas.

Agradecimentos. Agradecemos a Luis Gabriel Cuervo e Luis Alejandro Salicrup, do Escritório de Gestão do Conhecimento, Bioética e Pesquisa em Saúde, OPAS/OMS, e a Lisa Bero, da Universidade de Sydney.

Contribuição dos autores. TP é professor de medicina de família e pesquisador do sistema de saúde. JB trabalhou anteriormente como responsável pelas decisões no município de Piripiri, no nordeste do Brasil, e no Ministério da Saúde brasileiro como coordenador nacional 
da EVIPNet-Brasil. UP trabalhou anteriormente como funcionário sênior da OMS em Genebra, onde coordenou a Secretaria Global da EVIPNet. O artigo baseia-se em estudos de caso publicados em periódicos com revisão por pares, em documentos políticos e na experiência dos autores como pesquisadores e responsáveis por decisões em diferentes níveis do sistema de saúde. Todos os autores colaboraram neste artigo e concordam com seu conteúdo. TP é o garantidor. As opiniões externadas neste manuscrito são de responsabilidade do(s) autor(es) e não refletem necessariamente os critérios e políticas da RPSP / PAJPH e/ ou da OPAS/OMS.

Conflito de interesses. Lemos e compreendemos a política do BMJ sobre a declaração de interesses e não temos interesses relevantes a declarar.

Procedência do artigo e revisão por pares. Encomendado; revisão por pares externa.

Série Fortalecimiento da pesquisa em saúde nas Américas. Este artigo faz parte de uma série proposta pela OPAS e encomendada pelo BMJ, o qual foi responsável pela revisão por pares, edição e decisão de publicar o artigo, sem participação da OPAS. As despesas referentes à publicação desta série em Open Access foram financiadas pela OPAS. Para ler os outros artigos da série em inglês, visite www.bmj.com/health-research -americas; acesso à série em espanhol e português em https://www.paho .org/journal/es/numeros-especiales / fortalecimiento-investigacion-para -salud-americas

\section{REFERÊNCIAS}

1. The Mexico statement on health research: knowledge for better health: strengthening health systems. From the Ministerial Summit on Health Research. 2004. Disponível em inglês em: https://www.who.int/rpc /summit/agenda/en/mexico_statement on_health_research.pdf?ua $=1$.

2. Lancet. The Bamako call to action: research for health. Lancet 2008;372:1855. doi:10 .1016/S0140-6736(08)61789-4

3. Pan American Health Organization. Policy on research for health. 2009. Disponível em inglês em: http://www.paho.org/hq /images/stories/KBR/Research/research $\% 20$ policy $\% 20$ on $\% 20$ research $\% 20$ for $\% 20$ health\%20english.pdf.

4. Lavis JN, Lomas J, Hamid M, Sewankambo NK. Assessing country-level efforts to link research to action. Bull World Health Organ 2006;84:620-8. doi:10.2471/BLT.06.030312

5. Bennett S, Agyepong IA, Sheikh K, Hanson K, Ssengooba F, Gilson L. Building the field of health policy and systems research: an agenda for action. PLoS Med 2011;8:e1001081. doi:10.1371/journal.pmed .1001081

6. Oliver K, Innvar S, Lorenc T, Woodman J, Thomas J. A systematic review of barriers to and facilitators of the use of evidence by policymakers. BMC Health Serv Res 2014;14:2. doi:10.1186/1472-6963-14-2

7. Zacca-González G, Chinchilla-Rodríguez Z, Vargas-Quesada B, de Moya-Anegón F. Bibliometric analysis of regional Latin America's scientific output in Public Health through SCImago Journal \& Country Rank. BMC Public Health 2014;14:632. doi:10.1186/1471-2458-14-632

8. Reveiz L, Sangalang S, Glujovsky D, et al. Characteristics of randomized trials published in Latin America and the Caribbean according to funding source. PLoS One 2013;8:e56410. doi:10.1371/journal.pone .0056410

9. Becerra-Posada F, Minayo M, Quental C, de Haan S. National research for health systems in Latin America and the Caribbean: moving towards the right direction? Health Res Policy Syst 2014;12:13. doi:10 .1186/1478-4505-12-13

10. Organización Mundial de la Salud. $58^{\text {a }}$ Asamblea Mundial delaSalud: resoluciones y decisiones. 2005. Disponível em espanhol em: http://apps.who.int/gb/ebwha /pdf_files/WHA58-REC1/A58_2005_REC1 -sp.pdf.

11. Ellen ME, Lavis JN, Ouimet M, Grimshaw J, Bédard P-O. Determining research knowledge infrastructure for healthcare systems: a qualitative study. Implement Sci 2011;6:60. doi:10.1186/1748-5908-6-60

12. Chapman E. Management report and evaluation of EVIPNet Americas 20102013. Pan American Health Organization. 2013. Disponível em inglês em: https:// www.paho.org/hq/dmdocuments / 2015 /EVIPNetAmericas-Report-2010-2013 -ENG.pdf.

13. Entrevista con Oscar Ugarte Ubilluz, Ministro de Salud de Perú, acerca de políticas informadas en evidencia. 2011. Disponível em espanhol em: https:// www.paho.org/hq/index.php?option=com _content\&view $=$ article \&id $=5623: 2011$ -video-minister-health-peru-on-evidence -informed-policy-decision-making-spanish \&Itemid $=40301$ \&lang $=\mathrm{es}$

14. Gutierrez EL, Piazza M, Gutierrez-Aguado A, et al. Uso de la evidencia en políticas y programas de salud aportes del instituto nacional de salud [espanhol]. Rev Peru Med Exp Salud Publica 2016;33:580-4. doi:10.17843/rpmesp.2016.333.2308

15. Fondo Nacional de Investigacion en Salud. 10 años apoyando la investigación aplicada en salud en Chile [espanhol]. 2014. Disponível em espanhol em: https: / /www .conicyt.cl/fonis/files/2014/12/Fonis-10 -anios-de-apoyando-la-investigaci\%C3 $\% B 3 n-a p l i c a d a-e n-s a l u d-e n-C h i l e . p d f$.

16. Iglesias V, Erazo M, Droppelmann A, et al. Occupational second hand smoke is the main determinant of hair nicotine concentrations in bar and restaurant workers. Environ Res 2014;132:206-11. doi:10.1016 /j.envres.2014.03.044

17. Republica de Chile. Ministerio de Salud. Ley 20660: modifica ley no 19.419: en materia de ambientes libres de humo de tabaco. 2013. Disponível em espanhol em: https: / / www.leychile.cl/Navegar?idNorma $=1047848$.

18. World Health Organization. EVIPNet in action: 10 years, 10 stories. 2016. Dis- ponível em inglês em: https:/ /www.who.int /evidence/resources/publication/en/.

19. Moat KA, Lavis JN, Clancy SJ, El-Jardali F, Pantoja T. Knowledge Translation Platform Evaluation study team. Evidence briefs and deliberative dialogues: perceptions and intentions to act on what was learnt. Bull World Health Organ 2014;92:20-8. doi:10.2471/BLT.12.116806

20. Wichmann R, Carlan E, Barreto J. Consolidação da Rede para Políticas Informadas por Evidências - EVIPNet Brasil: relato da experiência nacional de construção de uma plataforma de tradução do conhecimento para o SUS. Bol Inst Saude 2016;17:13-25. Disponível em: http://www.saude.sp.gov.br /resources/instituto-de-saude/homepage /pdfs/bis17n1julho2016.pdf

21. Mansilla C, Herrera CA, Basagoitia A, Pantoja T. The evidence-informed policy network in Chile: lessons learned from a year of coordinated efforts. Rev Panam Salud Publica 2017;41:e36.

22. Erazo A. Un enfoque sistémico para comprender y mejorar los sistemas de salud [espanhol]. Rev Panam Salud Publica 2015;38:248-53.

23. El-Jardali F, Ataya N, Jamal D, Jaafar M. A multi-faceted approach to promote knowledge translation platforms in eastern Mediterranean countries: climate for evidence-informed policy. Health Res Policy Syst 2012;10:15. doi:10.1186/1478-4505-10-15

24. Ongolo-Zogo P, Lavis JN, Tomson G, Sewankambo NK. Climate for evidence informed health system policymaking in Cameroon and Uganda before and after the introduction of knowledge translation platforms: a structured review of governmental policy documents. Health Res Policy Syst 2015;13:2. doi:10.1186/1478 $-4505-13-2$

Como citar (artigo original): BMJ 2018;362:k2469. http:/ /dx.doi.org/10.1136/bmj.k2469

Manuscrito original em inglês publicado em 16 de julho de 2018. 